\title{
On the Interplay between Cannibalism and Harvest in Stage-Structured Population Models
}

\author{
Arild Wikan \\ Harstad University College, Havnegata 5, 9480 Harstad, Norway \\ Correspondence should be addressed to Arild Wikan; arild.wikan@hih.no
}

Received 23 March 2015; Accepted 3 June 2015

Academic Editor: Garth L. Fletcher

Copyright ( 2015 Arild Wikan. This is an open access article distributed under the Creative Commons Attribution License, which permits unrestricted use, distribution, and reproduction in any medium, provided the original work is properly cited.

\begin{abstract}
By use of a nonlinear stage-structured population model the role of cannibalism and the combined role of cannibalism and harvest have been explored. Regarding the model, we prove that in most parts of parameter space it is permanent. We also show that the transfer from stability to nonstationary dynamics always occurs when the unique stable equilibrium undergoes a supercritical Neimark-Sacker (Hopf) bifurcation. Moreover, the dynamic consequences of catch depend not only on which part of the population (immature or mature) is exposed to increased harvest pressure but also on which part of the immature population (newborns, older immature individuals) suffers from cannibalism. Indeed, if only newborns are exposed to cannibalism an enlargement of harvest pressure on the mature part of the population may act in a stabilizing fashion. On the other hand, whenever the whole immature population is exposed to cannibalism there are parts in parameter space where increased harvest on the mature population acts in a destabilizing fashion.
\end{abstract}

\section{Introduction}

Several species of commercial interest have been overexploited throughout the years. Among them we find salmon species like the capelin in the Barents Sea as well as several cod stocks around the world. Lots of other examples may be obtained in $[1,2]$. The global production of marine capture fisheries from around 19 million tonnes in catch in the 1950s has increased to around 80 million tonnes annually since the mid-1980s; see [3], and as documented in [4], there are species that have become extinct or almost extinct.

Another characteristic feature of lots of populations is that they oscillate. There may be a substantial difference in biomass from one year to another. Regarding fish populations there may be several causes for such fluctuations. One important factor is environmental changes, for example, changes in current systems which may have a crucial impact of newborns particularly; see [5] and references therein. The presence of one or several predator populations plays an important role too; see [6-8] or [9]. Internal factors like recruitment and cannibalism often act differently with respect to stability properties. While increased recruitment may give birth to chaotic oscillations, there is a tendency that increased cannibalism seems to stabilize the dynamics but not always (cf. the discussion in [10-14]). Finally, change in fishing patterns may also influence the dynamics as accounted for in $[9,15,16]$.

The purpose of this paper is to study the combined effects of recruitment, cannibalism, and harvest, and in doing so we apply a discrete stage-structured population model. In the next section we present the model and discuss its properties. An analysis of the impact of increasing recruitment and cannibalism is presented in Section 3. In Section 4 different harvest strategies are included as well in the model, and finally in Section 5 we summarize and discuss results.

\section{The Model}

At time $t$ we split the population $x_{t}$ into two separate parts, an immature part $x_{1, t}$ and a mature part $x_{2, t}$, and we further assume that the relation between the subpopulations at two consecutive time steps may be expressed by a system or difference equations:

$$
\begin{aligned}
& x_{1, t+1}=\left(F e^{-\beta_{1} x_{2, t}} x_{2, t}+\left(1-\mu_{1}\right) e^{-\beta_{2} x_{2, t}} x_{1, t}\right)\left(1-h_{1}\right), \\
& x_{2, t+1}=\left(\mu_{1} x_{1, t}+\left(1-\mu_{2}\right) x_{2, t}\right)\left(1-h_{2}\right)
\end{aligned}
$$


which we also may write on matrix form

$$
\underline{x}_{t+1}=A_{x} \underline{x}_{t},
$$

where $\underline{x}=\left(x_{1}, x_{2}\right)^{T}$ and

$$
A_{x}=\left(\begin{array}{cc}
\left(1-\mu_{1}\right)\left(1-h_{1}\right) e^{-\beta_{2} x_{2}} & F e^{-\beta_{1} x_{2}}\left(1-h_{1}\right) \\
\mu_{1}\left(1-h_{2}\right) & \left(1-\mu_{2}\right)\left(1-h_{2}\right)
\end{array}\right) .
$$

The meaning of the entries in (1) and (2) is as follows: $F, F>0$, is the fecundity, that is, the number of newborns per adult. $\mu_{1}$, $0<\mu_{1}<1$, is the fraction of the immature population that becomes mature one time unit later. $\left(1-\mu_{2}\right) x_{2, t}, 0<\mu_{2}<1$, is the part of the mature population which still lives one year later. The nonlinearities are of Ricker type and the parameters $\beta_{i}, \beta_{i} \geq 0, i=1,2$, will be referred to as cannibalism parameters. Consequently, the fecundity is reduced by the factor $e^{-\beta_{1} x_{2}}$ due to cannibalism from the mature population, and in the same way, the remaining part of the immature population $\left(1-\mu_{1}\right) x_{1}$ is reduced by a factor $e^{-\beta_{2} x_{2}}$. We assume no cannibalism pressure on $x_{2} . h_{1}$ and $h_{2}, 0 \leq h_{1}, h_{2} \leq 1$, are the fractions of each subpopulation which is removed through fishery, respectively.

Obviously, models (1) and (2) have a trivial equilibrium

$$
\left(\widetilde{x}_{1}, \tilde{x}_{2}\right)=(0,0) \text {. }
$$

Define the inherent net reproductive number $R_{0}$ as

$$
R_{0}=\frac{\left(1-h_{1}\right)\left(1-h_{2}\right) \mu_{1} F}{\left[1-\left(1-\mu_{1}\right)\left(1-h_{1}\right)\right]\left[1-\left(1-\mu_{2}\right)\left(1-h_{2}\right)\right]} .
$$

Then by stability analysis, it is straightforward to show that $\left(\widetilde{x}_{1}, \tilde{x}_{2}\right)$ is stable provided $R_{0}<1$. Therefore, we will in the rest of the paper assume that $R_{0} \geq 1$. Following [17], a population model is said to be permanent if there exist $\delta>0$ and $D>0$ such that

$$
\delta<\lim _{t \rightarrow \infty} \inf x_{t} \leq \lim _{t \rightarrow \infty} \sup x_{t}<D,
$$

where $x_{t}=x_{1, t}+x_{2, t}$ is the total population. Hence, if a population model is permanent the total population density neither explodes nor goes to zero. Regarding our models (1) and (2) we have the following result.

Theorem 1. Assume $R_{0}>1$. Then models (1) and (2) are permanent.

Proof. According to (6) we must show that the total population neither goes to zero (i) nor explodes (ii). Regarding (i), we have already shown that $R_{0}>1$ guarantees that the origin is a repellor. Moreover, the restriction on the parameters and functions given in (2) ensures that $A_{0}$ is irreducible and that $A_{x}$ is nonnegative for all $\left(x_{1}, x_{2}\right) \in \mathfrak{R}_{+}^{2}$. Consequently, (1) and (2) are $\mathfrak{R}_{+}^{2} \backslash\{0\}$ forward invariant. It remains to prove (ii) and in order to do that we need to show that there exists a compact set $X \subset \mathfrak{R}_{+}^{2}$ such that for all $\underline{x}_{0} \in \mathfrak{R}_{+}^{2}$ there exists $t^{M}=t^{M}\left(\underline{x}_{0}\right)$ satisfying $x_{t} \in X$ for all $t \geq t^{M}$. To this end, assume $\exp \left(-\beta_{1} x_{2, t}\right) x_{2, t} \leq K_{0}$, Then

$$
x_{1, t+1} \leq\left(1-\mu_{1}\right) x_{1, t}+F K_{0}
$$

and by induction

$$
x_{1, t} \leq\left(1-\mu_{1}\right)^{t} x_{1,0}+\frac{F K_{0}}{\mu_{1}} .
$$

Then there exists $t^{A}=t^{A}\left(x_{1,0}\right)$ such that for $t>t^{A}$

$$
x_{1, t+1} \leq \frac{2 F K_{0}}{\mu_{1}}=K_{1} \text {. }
$$

Further, in case of $t>t^{A},(1)$ and (2) also give

$$
x_{2, t+1} \leq \mu_{1} K_{1}+\left(1-\mu_{2}\right) x_{2, t}
$$

and once again (by induction) we find that for $t>t^{B}\left(x_{2,0}\right)$

$$
x_{2, t} \leq \frac{2 \mu_{1} K_{1}}{\mu_{2}}=K_{2} .
$$

Finally, take $t^{M}=\max \left\{t^{A}, t^{B}\right\}$ and $K=\max \left\{K_{1}, K_{2}\right\}$. Then, for $t \geq t^{M}, x_{1, t} \leq K, x_{2, t} \leq K$, and we are done.

Models (1) and (2) have also a nontrivial equilibrium $\left(x_{1}^{*}, x_{2}^{*}\right)$. Indeed, from (1)

$$
x_{1}^{*}=\frac{1-\left(1-\mu_{2}\right)\left(1-h_{2}\right)}{\mu_{1}\left(1-h_{2}\right)} x_{2}^{*}
$$

and $x_{2}^{*}$ is the solution of the equation $g\left(x_{2}\right)=0$, where

$$
\begin{aligned}
g\left(x_{2}\right)= & \frac{\mu_{1} F\left(1-h_{1}\right)\left(1-h_{2}\right)}{1-\left(1-\mu_{2}\right)\left(1-h_{2}\right)} e^{-\beta_{1} x_{2}} \\
& +\left(1-h_{1}\right)\left(1-\mu_{1}\right) e^{-\beta_{2} x_{2}}-1 .
\end{aligned}
$$

Clearly, $g^{\prime}\left(x_{2}\right)<0$ and in case of $x_{2}$ sufficiently large we conclude that $g\left(x_{2}\right)<0$. Moreover,

$$
\lim _{x_{2} \rightarrow 0} g\left(x_{2}\right)=\left(R_{0}-1\right)\left[1-\left(1-\mu_{1}\right)\left(1-h_{1}\right)\right]>0 .
$$

Hence, there exists $x_{2}=x_{2}^{*}$ such that $g\left(x_{2}^{*}\right)=0$. Consequently, the nontrivial equilibrium $\left(x_{1}^{*}, x_{2}^{*}\right)$ is unique.

Let $J$ be the Jacobian of (1) and (2) evaluated at $\left(x_{1}^{*}, x_{2}^{*}\right)$. Then $\left(x_{1}^{*}, x_{2}^{*}\right)$ is stable whenever the following inequalities hold:

$$
\begin{array}{r}
1-\operatorname{tr} J+|J|>0, \\
1+\operatorname{tr} J+|J|>0, \\
1-|J|>0 .
\end{array}
$$


After some time-consuming calculations it is possible to rewrite (15a), (15b), and (15c) as

$$
\begin{aligned}
& {[}\left.1-\left(1-\mu_{2}\right)\left(1-h_{2}\right)\right] \\
& \cdot {\left[\beta_{1}-\left(1-\mu_{1}\right)\left(1-h_{1}\right)\left(\beta_{1}-\beta_{2}\right) e^{-\beta_{2} x_{2}^{*}}\right] x_{2}^{*}>0, } \\
& 2\left(1-\mu_{2}\right)\left(1-h_{2}\right)+\left[1-\left(1-\mu_{2}\right)\left(1-h_{2}\right)\right] \beta_{1} x_{2}^{*} \\
& \quad+\left(1-\mu_{1}\right)\left(1-h_{1}\right) \\
& \cdot\left[2-\left[1-\left(1-\mu_{2}\right)\left(1-h_{2}\right)\right]\left(\beta_{1}-\beta_{2}\right) x_{2}^{*}\right] e^{-\beta_{2} x_{2}^{*}} \\
& \quad>0, \\
& 2-\left(1-\mu_{2}\right)\left(1-h_{2}\right)-\left[1-\left(1-\mu_{2}\right)\left(1-h_{2}\right)\right] \beta_{1} x_{2}^{*} \\
&-e^{-\beta_{2} x_{2}^{*}}\left(1-h_{1}\right)\left(1-\mu_{1}\right) \\
& \cdot\left\{1-\left[1-\left(1-\mu_{2}\right)\left(1-h_{2}\right)\right]\left(\beta_{1}-\beta_{2}\right)\right\} x_{2}^{*}>0 .
\end{aligned}
$$

If both eigenvalues $\lambda$ of the linearization of (1) and (2) are located inside the unit circle in the complex plane, (16a), (16b), and (16c) hold and $\left(x_{1}^{*}, x_{2}^{*}\right)$ is stable. The left hand side of (16a) fails to be positive when an eigenvalue crosses the unit circle through 1 and a saddle-node bifurcation occurs. (16b) fails when $\lambda=-1$. This gives birth to a flip (period doubling) bifurcation; hence when $\left(x_{1}^{*}, x_{2}^{*}\right)$ fails to be stable the result is a 2-period orbit. (16c) fails when $\lambda$ becomes a complex number located on the boundary of the unit circle. In this case the equilibrium will undergo a NeimarkSacker (Hopf) bifurcation at instability threshold, and as we penetrate into the unstable parameter region, quasiperiodic orbits restricted to an invariant curve will be the outcome provided the bifurcation is of supercritical type.

\section{Recruitment and Cannibalism}

First, let us focus on the dynamics in case of no harvest (i.e., $\left.h_{1}=h_{2}=0\right)$.

(1) Assume $\beta_{1} \neq 0, \beta_{2}=0$ which means that only newborns are exposed to cannibalism.

Then $R_{0}=F / \mu_{2}$ and the nontrivial equilibrium becomes

$$
\left(x_{1}^{*}, x_{2}^{*}\right)=\left(\frac{\mu_{2}}{\mu_{1}} \frac{1}{\beta_{1}} \ln R_{0}, \frac{1}{\beta_{1}} \ln R_{0}\right) .
$$

Moreover, criteria (16a) and (16b) degenerate to $\mu_{1} \mu_{2} \ln R_{0}>$ $0,2\left(2-\mu_{1}-\mu_{2}\right)+\left(2-\mu_{1}\right) \mu_{2} \ln R_{0}>0$, respectively, and both of them are obviously valid. Inequality (16c) may be expressed as

$$
R_{0}<\exp \left(\frac{\mu_{1}+\mu_{2}}{\mu_{1} \mu_{2}}\right) .
$$

Hence, $\left(x_{1}^{*}, x_{2}^{*}\right)$ fails to be stable when (18) becomes an equality and a Neimark-Sacker bifurcation occurs.

As is well known bifurcations may be of both supercritical and subcritical nature. If a fixed point will undergo a supercritical bifurcation it means that an eigenvalue $\lambda$ must cross the unit circle outwards at instability and in the Neimark-Sacker case that an attracting (stable) quasiperiodic orbit restricted on an invariant curve is created beyond the threshold.

Now, considering (1) and (2) we increase $R_{0}$ through an increase of $F$. Thus the bifurcation takes place at $F=F_{T}=$ $\mu_{2} \exp \left(\left(\mu_{1}+\mu_{2}\right) /\left(\mu_{1} \mu_{2}\right)\right)$. Moreover, the eigenvalues may be expressed as

$$
\begin{aligned}
\lambda= & \frac{1}{2}\left\{2-\mu_{1}-\mu_{2}\right. \\
& \left. \pm \sqrt{4\left[\left(1-\mu_{1}\right)\left(1-\mu_{2}\right)-\mu_{1} \mu_{2}\left(1-\beta_{1} x_{2}^{*}\right)\right]-\left(2-\mu_{1}-\mu_{2}\right)^{2}} i\right\}
\end{aligned}
$$

and an easy computation shows that

$$
\frac{d}{d F}|\lambda|=\frac{1}{2 \mu_{1}} e^{-\left(\mu_{1}+\mu_{2}\right) / \mu_{1} \mu_{2}}>0
$$

(evaluated at $F=F_{T}$ ) which proves that $\lambda$ leaves the unit circle at threshold.

In order to show that the quasiperiodic orbit is stable when $F>F_{T},|F-F T|$ small, we first write (1) and (2) on complex form and then through a series of near identity transformations (normal form calculations) formally express it as

$$
z \longrightarrow z e^{i \theta_{0}}\left(1+F+d|z|^{2}\right)+O\left(|z|^{4}\right)
$$

(for details, cf. [18] or [19]) and the sign of $a=\operatorname{Re}(d)$ will determine the nature of bifurcation, $a<0$ implies supercritical, and $a>0$ implies subcritical.

Regarding (1) and (2) we may partly rest upon findings obtained in [13] and express $a$ as

$$
\begin{aligned}
a= & -\frac{\beta_{1}^{2}}{16\left(\mu_{1}+\mu_{2}\right)}\left\{\left(2 \mu_{1} \mu_{2}\right)^{2}\right. \\
& \left.+\left(\mu_{1}+\mu_{2}\right)\left[\left(2 \mu_{1} \mu_{2}-\left(\mu_{1}+\mu_{2}\right)\right)^{2}-\mu_{1} \mu_{2}\right]\right\}
\end{aligned}
$$

which is negative whenever $\beta_{1}>0,0<\mu_{1}, \mu_{2}<1$. Consequently, the bifurcation is supercritical.

In order to visualize the findings above we show in Figure 1(a) an orbit which converges towards the stable equilibrium $\left(x_{1}^{*}, x_{2}^{*}\right), R_{0}<\exp \left(\left(\mu_{1}+\mu_{2}\right) / \mu_{1} \mu_{2}\right)$. Figure $1(\mathrm{~b})$ shows the situation after the supercritical bifurcation $R_{0}>$ $\exp \left(\left(\mu_{1}+\mu_{2}\right) / \mu_{1} \mu_{2}\right)$. The dynamics is restricted to an attracting invariant curve and on that curve (1) and (2) act as a circle map which rotates points around the curve with an irrational winding number. This scenario persists in a large $R_{0}$ interval but eventually chaos is introduced when the curve starts to break up. This is displayed in Figure 1(c).

(2) Next, assume $\beta_{1}=\beta_{2}=\beta$ (same cannibalism pressure on newborns and older immature individuals).

In this case

$$
\left(x_{1}^{*}, x_{2}^{*}\right)=\left(\frac{\mu_{2}}{\mu_{1}} \frac{1}{\beta} \ln K, \frac{1}{\beta} \ln K\right),
$$

where $K=\mu_{1}\left(R_{0}-1\right)+1$ and $R_{0}=F / \mu_{2}$ and we notice that $K^{\prime}\left(R_{0}\right)>0$. 


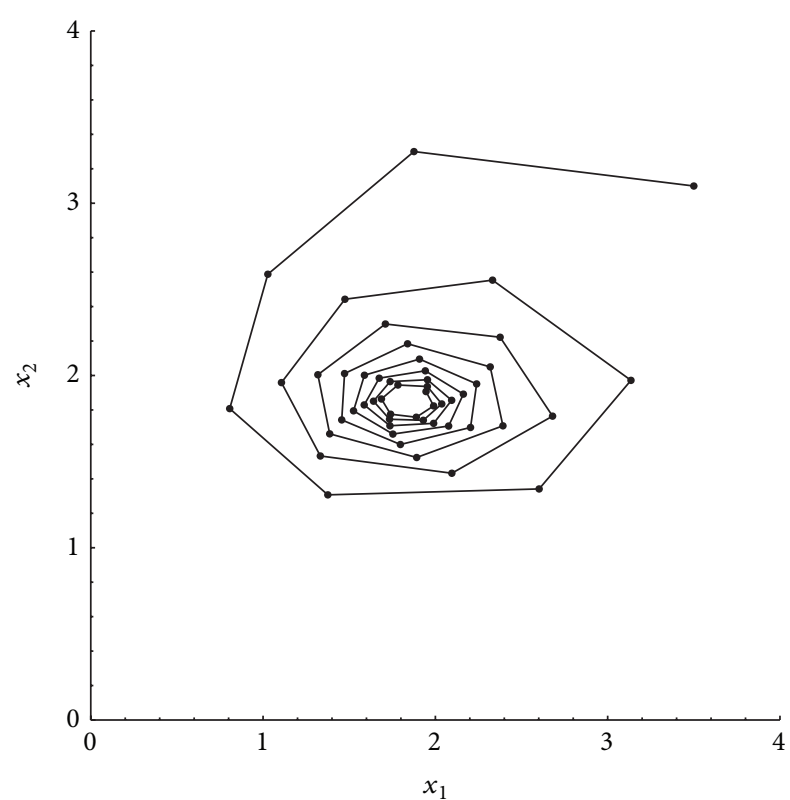

(a)

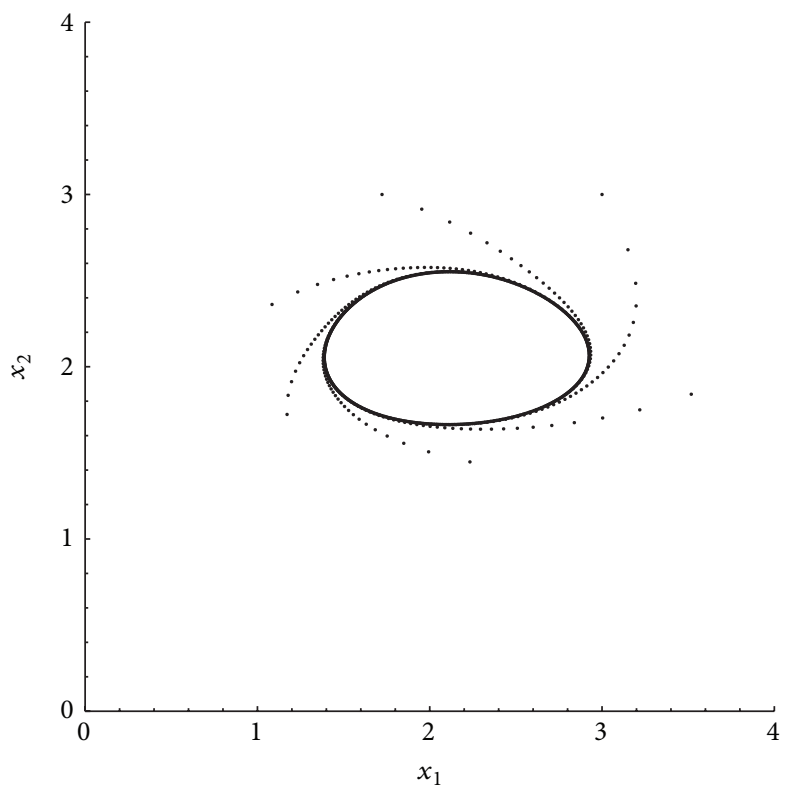

(b)

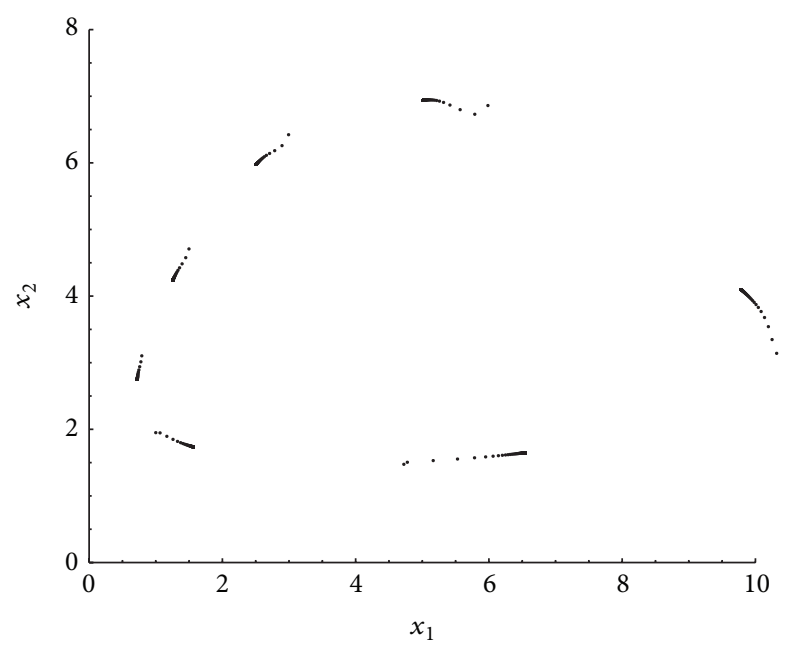

(c)

Figure 1: (a) An orbit converging towards the stable equilibrium. Parameter values $\beta_{1}=2, \beta_{2}=0, F=20$, and $\mu_{1}=\mu_{2}=0.5$. (b) A stable attractor generated by (1) and (2). Parameter values as in (a) except $F=30$. (c) The invariant curve has broken up. Parameter values as in (a) and (b) except $F=107$.

Stability criteria (16a) and (16b) reduce to $\mu_{2} \ln K>0$, $2\left(1-\mu_{2}\right)+\mu_{2} \ln K+2\left(1-\mu_{1}\right) K^{-1}>0$, respectively, and both of them clearly hold. Criterion (16c) may be written as

$$
\mu_{2} \ln K+\frac{1-\mu_{1}}{K}<1+\mu_{2} \text {. }
$$

Obviously, $R_{0} \rightarrow 1^{+}$implies that the left hand side of (24) is less than the right hand side but as $R_{0}$ increases there must exists a threshold where a (supercritical) Neimark-Sacker bifurcation occurs. Thus, qualitatively, we have arrived at the same situation as in the $\beta_{1} \neq 0, \beta_{2}=0$ case.

Beyond instability we observe quasiperiodic orbits restricted to an invariant curve (cf. Figure 2(a)). As we continue to increase $R_{0}$ the curve becomes kinked (Figure 2(b)) and therefore not topologically equivalent to a circle anymore. Eventually, the curve breaks up here too. However, note that we use the same $F$ value when we generated Figures 1(c) and 2(b). Hence, one may argue that if both newborns and older immature individuals are exposed to cannibalism stability properties are improved compared to the case where $\beta_{2}=0$.

(3) The third case to discuss is $\beta_{1}=0, \beta_{2} \neq 0$ which biologically means that newborns are not exposed to cannibalism from older mature relatives but must reach a certain size (or age) to serve as food. From Theorem 1 we cannot conclude that (1) and (2) are a permanent population 


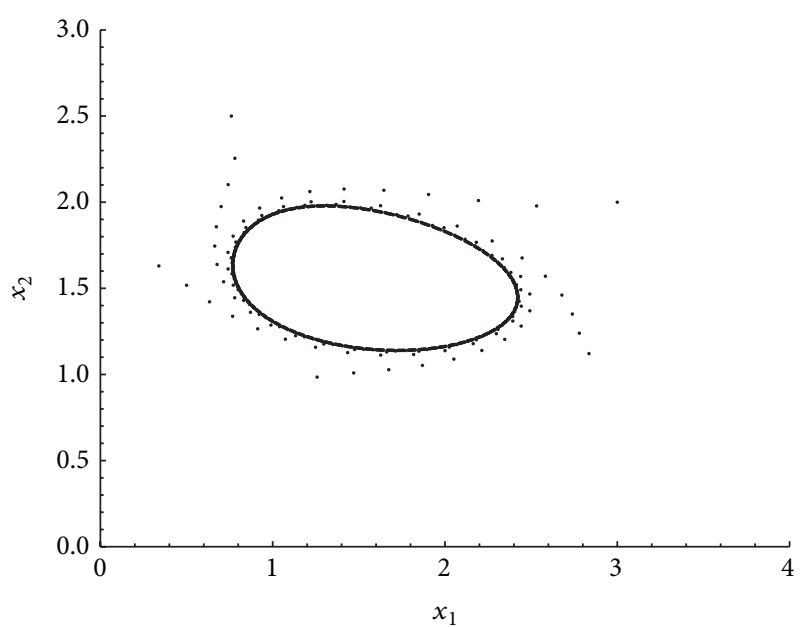

(a)

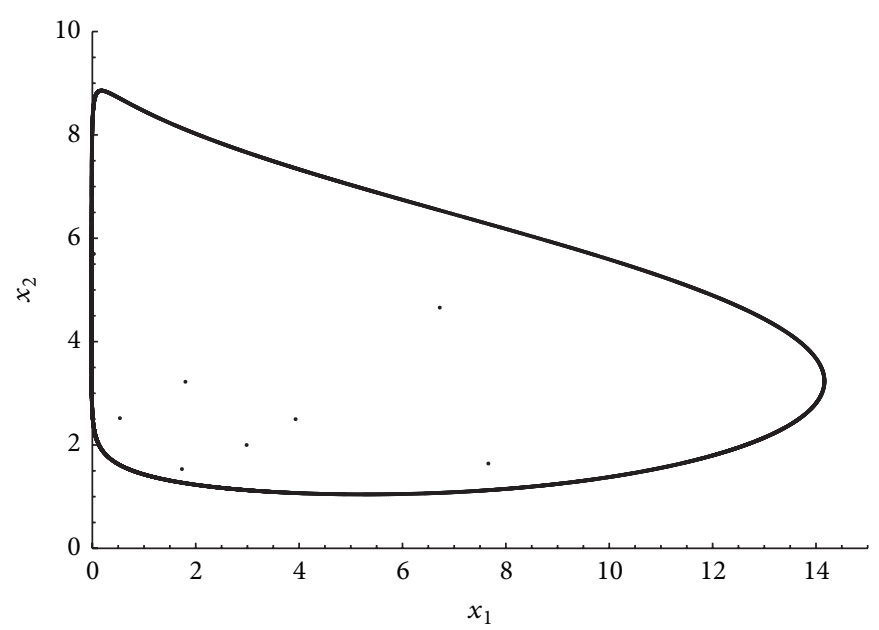

(b)

Figure 2: (a) An invariant curve just beyond instability threshold. Parameter values $\beta_{1}=\beta_{2}=2, \mu_{1}=\mu_{2}=0.5$, and $F=20$. (b) The attractor when $F=107$. Other parameter values as in (a).

model. There is simply too little density dependence. The equilibrium point

$$
\begin{aligned}
& \left(x_{1}^{*}, x_{2}^{*}\right) \\
& \quad=\left(\frac{\mu_{2}}{\mu_{1}} \frac{1}{\beta_{2}} \ln \left(\frac{1-\mu_{1}}{1-\mu_{1} R_{0}}\right), \frac{1}{\beta_{2}} \ln \left(\frac{1-\mu_{1}}{1-\mu_{1} R_{0}}\right)\right)
\end{aligned}
$$

exists and is stable only in the tiny interval $1<R_{0}<1 / \mu_{1}$. If $R_{0}>1 / \mu_{1}$ the population goes to infinity. Note that such a phenomenon is by no means unique. It has been detected in other nonlinear models as well; see, for example, [20] or the prey-predator model in [8]. When $R_{0}>1 / \mu_{1}$ models (1) and (2) behave more or less as a linear model as accounted for in [21]. Also, compare [22].

\section{Cannibalism and Harvest}

Let us now focus on the role of harvest and the combined effect of cannibalism and harvest with respect to stability. We concentrate on cases where (1) and (2) are a permanent population model and we restrict the analysis to (i) $h_{1}=$ $0, h_{2} \neq 0$, (ii) $h_{1} \neq 0, h_{2}=0$, and (iii) $h_{1}=h_{2}=$ $h \neq 0$. Under these restrictions we have computed equilibria and stability conditions and the results are presented in Table 1 . The various stability criteria are found from (16c). Our analysis shows that (16a) and (16b) always hold.

We start by considering the case of $\beta_{1} \neq 0$ and $\beta_{2}=0$ which is covered by (A), (B), and (C) in Table 1 .

If $\mu_{1}<\mu_{2}$ and $h_{1}=0$ and $h_{2} \neq 0$ numerical experiments show that the size of the total equilibrium population at instability threshold is larger than in case of no harvest. A natural way to implement this is to say that an increase of harvest of the mature population leads to better stability properties of the whole population (the size of the mature equilibrium population is reduced while the size of the immature population is increased). On the other hand, $h_{1} \neq 0$ and $h_{2}=0$ result in a smaller equilibrium population at instability threshold compared to the case of no harvest. Hence, harvest acts in a destabilizing fashion. If we apply the same harvest pressure $h\left(h=h_{1}=\right.$ $h_{2}$ ) on the subpopulations we experience that $h$ acts as a weak destabilizing effect which means that the destabilizing effect of harvesting fractions of the immature population is somewhat stronger than the stabilizing effect of harvesting fractions of the mature population.

Turning to the case $\mu_{1}=\mu_{2}$ much of the same picture emerges. An increase of $h_{2}$ stabilizes while an increase of $h_{1}$ destabilizes. The difference between this case and $\mu_{1}<\mu_{2}$ is that the effect from harvesting the mature population now dominates. Thus, an increase of $h$ turns out to be a (weak) stabilizing effect. Finally, if $\mu_{1}>\mu_{2}$ we find (as before) that an increase of $h_{1}$ acts in destabilizing fashion and that an increase of $h_{2}$ acts in stabilizing fashion. The combined effect of $h_{1}$ and $h_{2}$ acts in a weak destabilizing way. Hence, the overall conclusion from the cases discussed above is that an increase of $h_{1}$ is a destabilizing effect while an increase of $h_{2}$ tends to stabilize the dynamics. The effect of increasing $h$ $\left(h=h_{1}=h_{2}\right)$ depends on $\mu_{1}$ and $\mu_{2}$.

Let us now consider the cases where not only newborns but also immature relatives are exposed to cannibalism from the mature population. These cases are covered by (D), (E), and (F) in Table 1. Assuming $\mu_{1}<\mu_{2}$ we have by use of (D) adjusted $F$ such that the dynamics in absence of harvest is restricted to an invariant curve (numerical example, $\mu_{1}=0.3$, $\mu_{2}=0.7$, and $F=23$ ). Now, keeping $h_{2}=0$, an increase of $h_{1}$ makes the invariant curve disappear and we observe a weakly attracting fixed point. The same qualitative picture emerges both when $h_{1}=0$ and we increase $h_{2}$ and also when $h_{1}=h_{2}=$ $h$ is increased. Thus, by use of (D), (E), and (F) and numerical experiments we conclude that harvest acts in a stabilizing fashion. Moreover, we experience the same situation under the assumption $\mu_{1}=\mu_{2}$. This is displayed in Figures 3(a) and 3(b). In Figure 3(a) (no harvest) map (1) generates an orbit restricted to an invariant curve. In Figure 3(b) where 


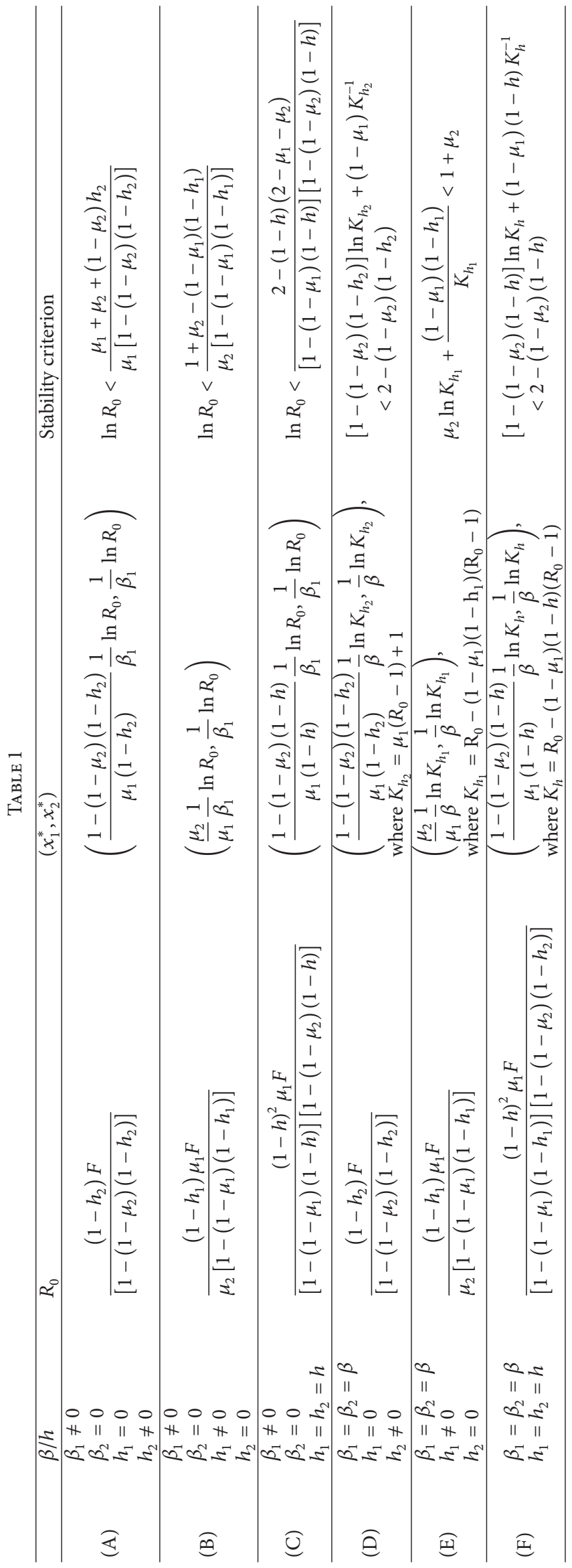




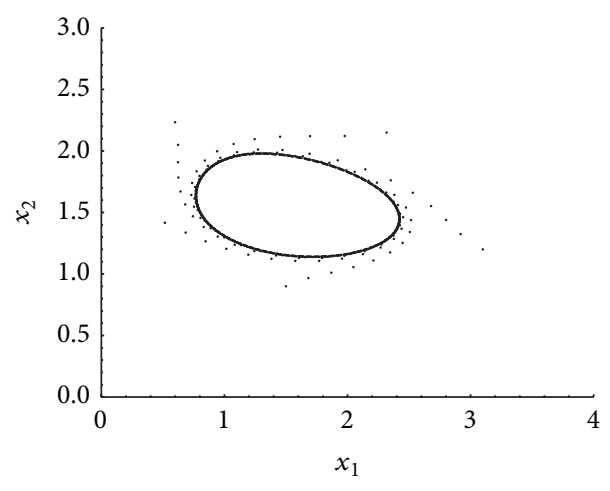

(a)

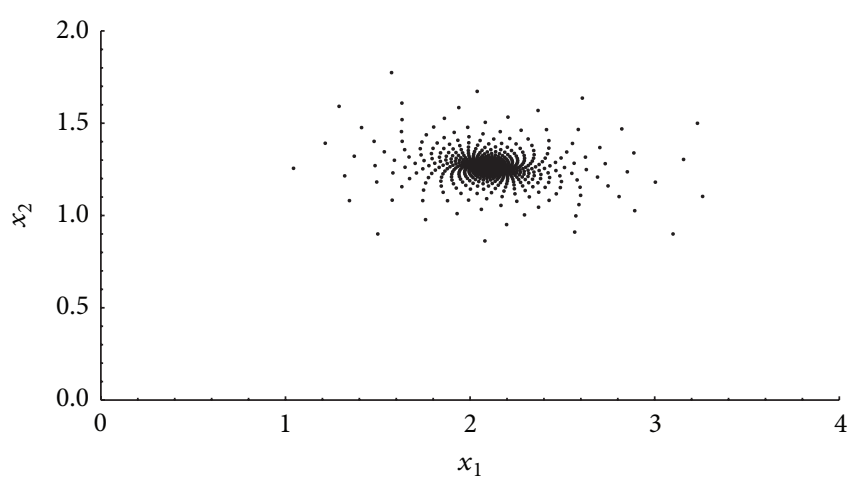

(b)

Figure 3: (a) An invariant curve generated by (1) and (2). Parameter values $\mu_{1}=\mu_{2}=0.5, F=20$, and $h_{1}=h_{2}=0$. (b) An attracting fixed point. Parameter values as in (a) but $h_{2}=0.25$.

$h_{2}=0.25$ we see a weakly stable fixed point together with some initial transients.

The final case to discuss is $\mu_{1}>\mu_{2}$ and again, in the absence of harvest, it is possible to obtain nonstationary dynamics provided $F$ is sufficiently large. If $h_{1}=0$ an enlargement of $h_{2}$ leads to a "larger radius" of the invariant curve; hence we observe the same picture as earlier but the population is located further away from the fixed point. Consequently, it is natural to classify an increase of $h_{2}$ as a destabilizing factor. On the other hand, an increase of $h_{1}$ $\left(h_{2}=0\right)$ acts in stabilizing fashion in the same way as in the $\mu_{1}<\mu_{2}$ and $\mu_{1}=\mu_{2}$ cases. The combined effect of increasing both $h_{1}$ and $h_{2}$ turns out to be destabilizing (in a rather weak fashion).

\section{Summary and Discussion}

By use of a stage-structured population model, the purpose of this paper has been to reveal the dynamical consequences of cannibalism and the combined consequences of cannibalism and harvest. Despite the fact that there are two stages only (immature and mature) the parameter space is huge so some care should be taken with respect of drawing too strong conclusions.

Considering the effect of cannibalism alone, in the cases $\beta_{1} \neq 0, \beta_{2}=0$, or $\beta=\beta_{1}=\beta_{2}$, stability criteria are given by (18) and (24), respectively. Here we may notice that neither (18) nor (24) contains any cannibalism parameters which means that the values of $\beta_{i}$ contribute to the size of the equilibrium population only, not to qualitative changes of the dynamics. Nonstationary dynamics is achieved through an enlargement of $R_{0}$ (or $F$ ). If only newborns are exposed to cannibalism it is possible to generate nonstationary as well as chaotic dynamics. When both $\beta_{i}$ and $\beta_{2} \neq 0$ the same qualitative picture is established but this implies much larger $R_{0}$ values. Thus a natural interpretation is to say that the more the immature population suffers from cannibalism, the better the stability properties of the population as a whole are.

Now, assuming that the population is exposed to both cannibalism and harvest, let us first comment on the situation where $\beta_{1} \neq 0, \beta_{2}=0$. Then the value of $R_{0}$ and stability conditions are given by (A), (B), and (C) in Table 1 and in all cases $\left(\mu_{1}<\mu_{2}, \mu_{1}=\mu_{2}, \mu_{1}>\mu_{2}\right)$ our finding is that an increase of harvest $h_{1}$ of the immature part of the population acts in a destabilizing fashion while an enlargement of $h_{2}$ improves stability properties. The effect which is the most profound one depends on $\mu_{1}, \mu_{2}$.

If both newborns and older immature relatives are exposed to cannibalism $\left(\beta=\beta_{1}=\beta_{2} \neq 0\right)$ stability criteria and $R_{0}$ are given by (D), (E), and (F) in Table 1 . As is shown, the results obtained here are in many respects quite different from the case $\beta_{2}=0$. Now, $\mu_{1} \leq \mu_{2}$ implies an increase of both $h_{1}$ and $h_{2}$ that act as stabilizing factors. If $\mu_{1}>\mu_{2}$ then an increase of $h_{2}$ turns out to improve stability properties. Thus, what these results show is that the impact of harvest may act differently depending on which part of the immature population is exposed to cannibalism.

It is tempting to compare findings above with results obtained from analysis of age-structured Leslie matrix population models, that is, models on the form

$$
\begin{gathered}
x_{1, t+1}=\left(\sum_{i=1}^{n} F e^{-x_{t}} x_{i, t}\right)\left(1-h_{1}\right), \\
x_{2, t+1}=P_{1} x_{1, t}\left(1-h_{2}\right), \\
\vdots \\
x_{n, t+1}=P_{n-1} x_{n-1, t}\left(1-h_{n}\right) .
\end{gathered}
$$

Model (26) consists of $\mathrm{n}$ nonoverlapping age classes $x_{1}, \ldots, x_{n}$ and $x_{t}=\sum x_{i}$ is the total population. Moreover, $F$ and $h_{i}$ have the same meaning as in (1) and $P_{i}, 0<P_{i} \leq 1$, are the year to year survival probabilities. Observe that, in case of $n$ large, individuals spend most of their lives as grownups. In particular this is the case when the year to year survival probabilities are close to unity. This corresponds to $\mu_{1}>\mu_{2}$ in (1). (Indeed, if $\mu_{1}$ is large, then individuals spend only a tiny part of their lives as juveniles and if $\mu_{2}$ is small then the 
"grownup part" is large.) Note that (1) and (26) rest upon somewhat different prerequisites as accounted for in [22].

Now, considering (26), in the absence of harvest we find that an increase of the number of age classes turns out to be a stabilizing effect (cf. [23]). Harvest acts more and more in destabilizing fashion as $n$ is increased, especially when the survival probabilities become large. Hence, in the interplay between increasing the number of age classes and harvest the destabilizing effect of the latter turns out to be the dominant one. Thus, in many respects, this is consistent with the qualitative findings from (1) in the $\mu_{1}>\mu_{2}$ case.

\section{Conflict of Interests}

The author declares that there is no conflict of interests regarding the publication of this paper.

\section{References}

[1] FAO, The State of World Fisheries and Aquaculture, Food and Agriculture Organization, Rome, Italy, 1st edition, 2004.

[2] T. E. E. Oldfield, R. J. Smith, S. R. Harrop, and N. LeaderWilliams, "A gap analysis of terrestrial protected areas in England and its implications for conservation policy," Biological Conservation, vol. 120, no. 3, pp. 303-309, 2004.

[3] S. Tang and L. Chen, "The effect of seasonal harvesting on stage-structured population models," Journal of Mathematical Biology, vol. 48, no. 4, pp. 357-374, 2004.

[4] R. A. Myers, J. A. Hutchings, and N. J. Barrowman, "Why do fish stocks collapse? The example of cod in Atlantic Canada," Ecological Applications, vol. 7, no. 1, pp. 91-106, 1997.

[5] A. Wikan, "On reserves, stability and the maximum sustainable yield problem," Journal of Mathematics and Statistics, vol. 9, no. 4, pp. 325-333, 2013.

[6] M. G. Neubert and M. Kot, "The subcritical collapse of predator populations in discrete-time predator-prey models," Mathematical Biosciences, vol. 110, no. 1, pp. 45-66, 1992.

[7] B. Bogstad and S. Mehl, "Interactions between Atlantic Cod (Gadus morhua) and its prey species in the Barents Sea," in Proceedings of the Forage Fishes in Marine Ecosystems (FME '97), Alaska Sea Grant College Program, 1997.

[8] A. Wikan, "From chaos to chaos. An analysis of a discrete age-structured prey-predator model," Journal of Mathematical Biology, vol. 43, no. 6, pp. 471-500, 2001.

[9] O. Flaaten and E. Mjølhus, "Using reserves to protect fish and wildlife-simplified modeling approaches," Natural Resource Modeling, vol. 18, no. 2, pp. 157-182, 2005.

[10] J. M. Cushing, "A simple model of cannibalism," Mathematical Biosciences, vol. 107, no. 1, pp. 47-71, 1991.

[11] F. Van den Bosch and W. Gabriel, "Cannibalism in an agestructured predator-prey system," Bulletin of Mathematical Biology, vol. 59, no. 3, pp. 551-567, 1997.

[12] K. G. Magnússon, "Destabilizing effect of cannibalism on a structured predator-prey system," Mathematical Biosciences, vol. 155, no. 1, pp. 61-75, 1999.

[13] A. Wikan and A. Eide, "An analysis of a nonlinear stagestructured cannibalism model with application to the Northeast Arctic cod stock," Bulletin of Mathematical Biology, vol. 66, no. 6, pp. 1685-1704, 2004.
[14] A. Eide and A. Wikan, "Optimal selection and effort in a fishery on a stock with cannibalistic behaviour the case of the northeast arctic cod fisheries," Journal of Fisheries and Aquatic Science, vol. 5, no. 6, pp. 454-468, 2010.

[15] A. Hastings and L. W. Botsford, "Equivalence in yield from marine reserves and traditional fisheries management," Science, vol. 284, no. 5419, pp. 1537-1538, 1999.

[16] C. W. Armstrong and U. R. Sumaila, "Cannibalism and the optimal sharing of the North-East Atlantic cod stock: a bioeconomic model," Journal of Bioeconomics, vol. 2, no. 2, pp. 99-115, 2000.

[17] R. Kon, Y. Saito, and Y. Takeuchi, "Permanence of single-species stage-structured models," Journal of Mathematical Biology, vol. 48, no. 5, pp. 515-528, 2004.

[18] J. Guckenheimer and P. Holmes, Nonlinear Oscillations, Dynamical Systems and Bifurcations of Vector Fields, Springer, New York, NY, USA, 1990.

[19] W. Govaerts and R. K. Ghaziani, "Numerical bifurcation analysis of a nonlinear stage structured cannibalism population model," Journal of Difference Equations and Applications, vol. 12, no. 10, pp. 1069-1085, 2006.

[20] A. Wikan and E. Mjølhus, "Overcompensatory recruitment and generation delay in discrete age-structured population models," Journal of Mathematical Biology, vol. 35, no. 2, pp. 195-239, 1996.

[21] P. Cull and A. Vogt, "Mathematical analysis of the asymptotic behavior of the Leslie population matrix model," Bulletin of Mathematical Biology, vol. 35, pp. 645-661, 1973.

[22] H. Caswell, Matrix Population Models, Sinauer Associates, Sunderland, Mass, USA, 2nd edition, 2001.

[23] A. Wikan, "Dynamical consequences of harvest in discrete age-structured population models," Journal of Mathematical Biology, vol. 49, no. 1, pp. 35-55, 2004. 

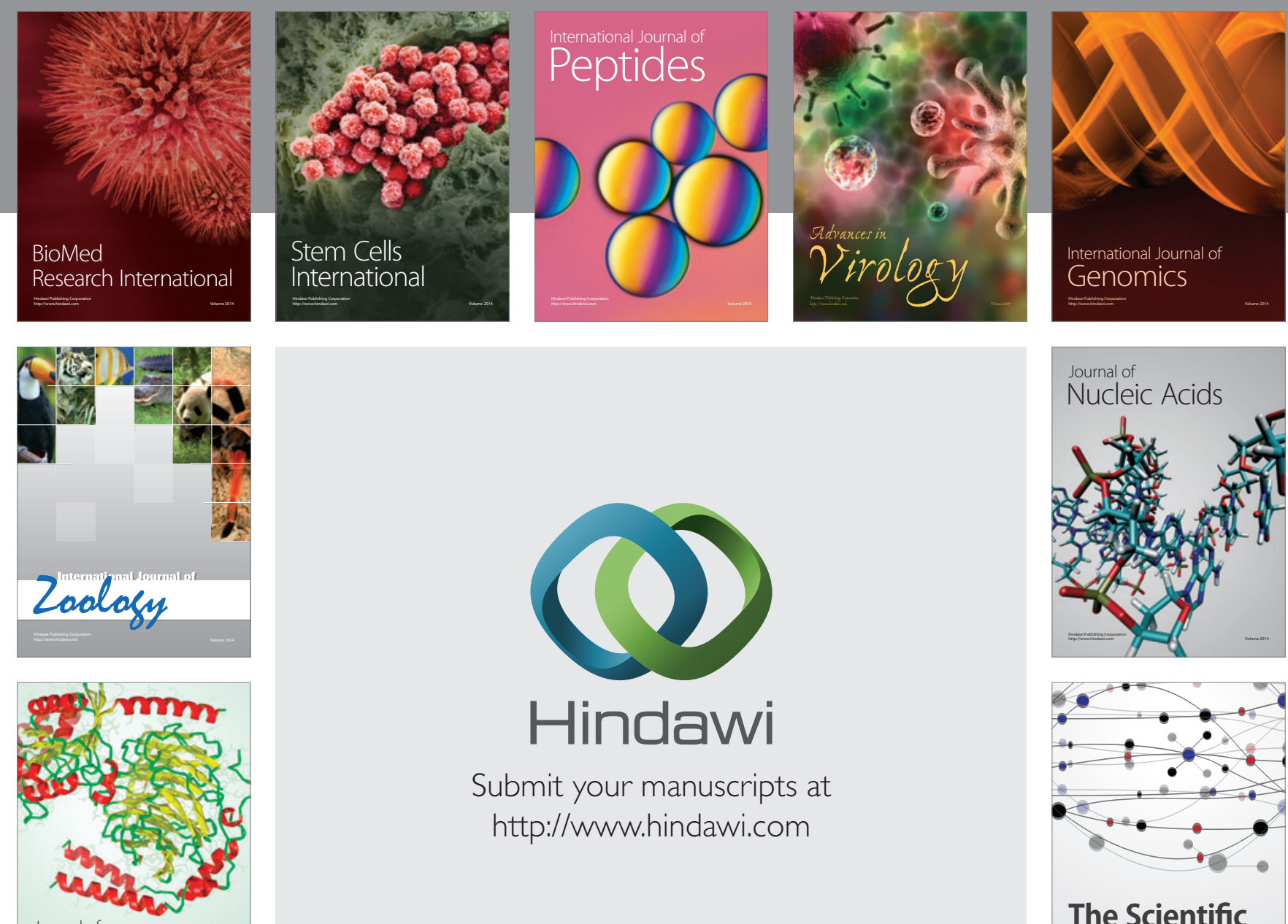

Submit your manuscripts at

http://www.hindawi.com

Journal of
Signal Transduction
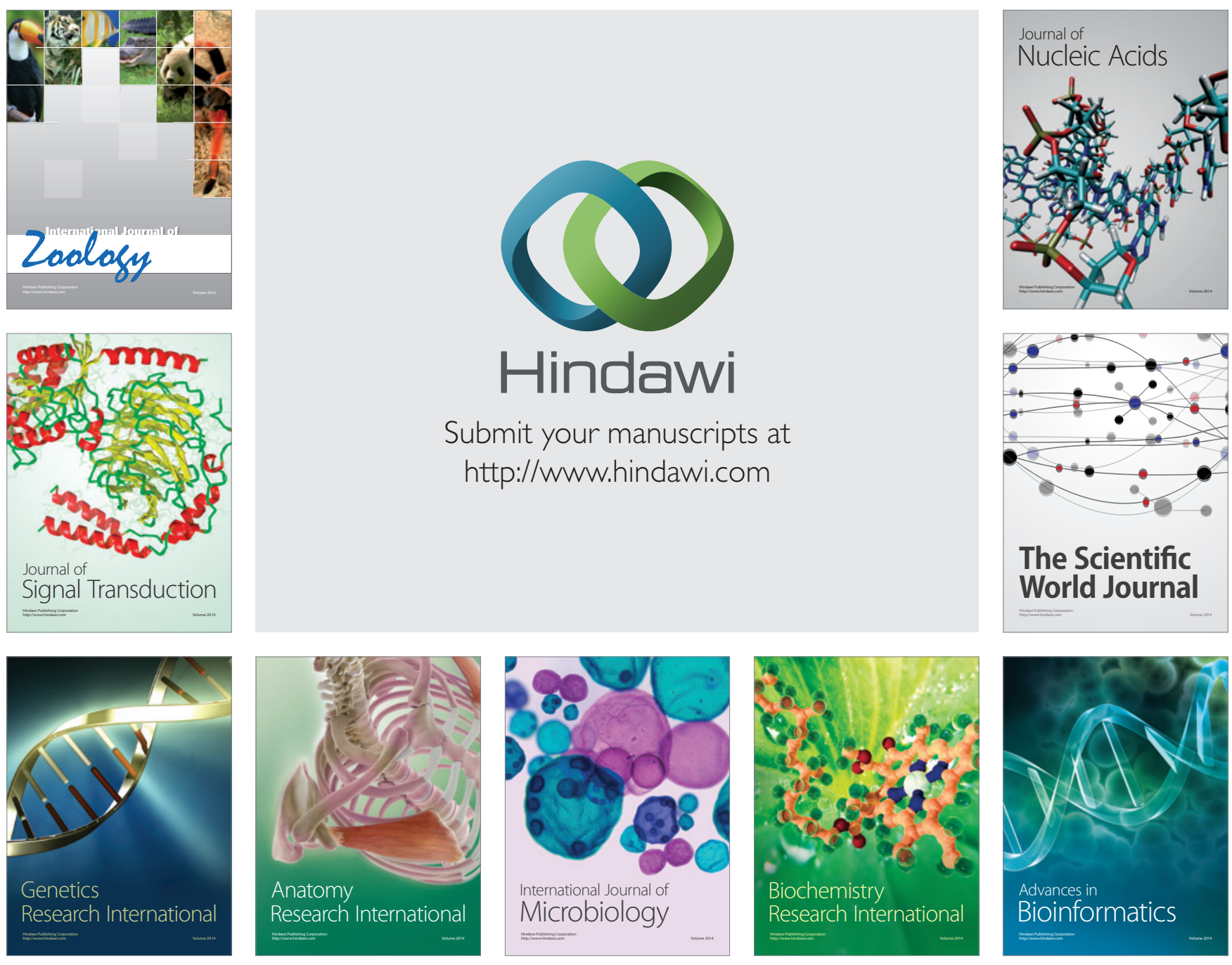

The Scientific World Journal
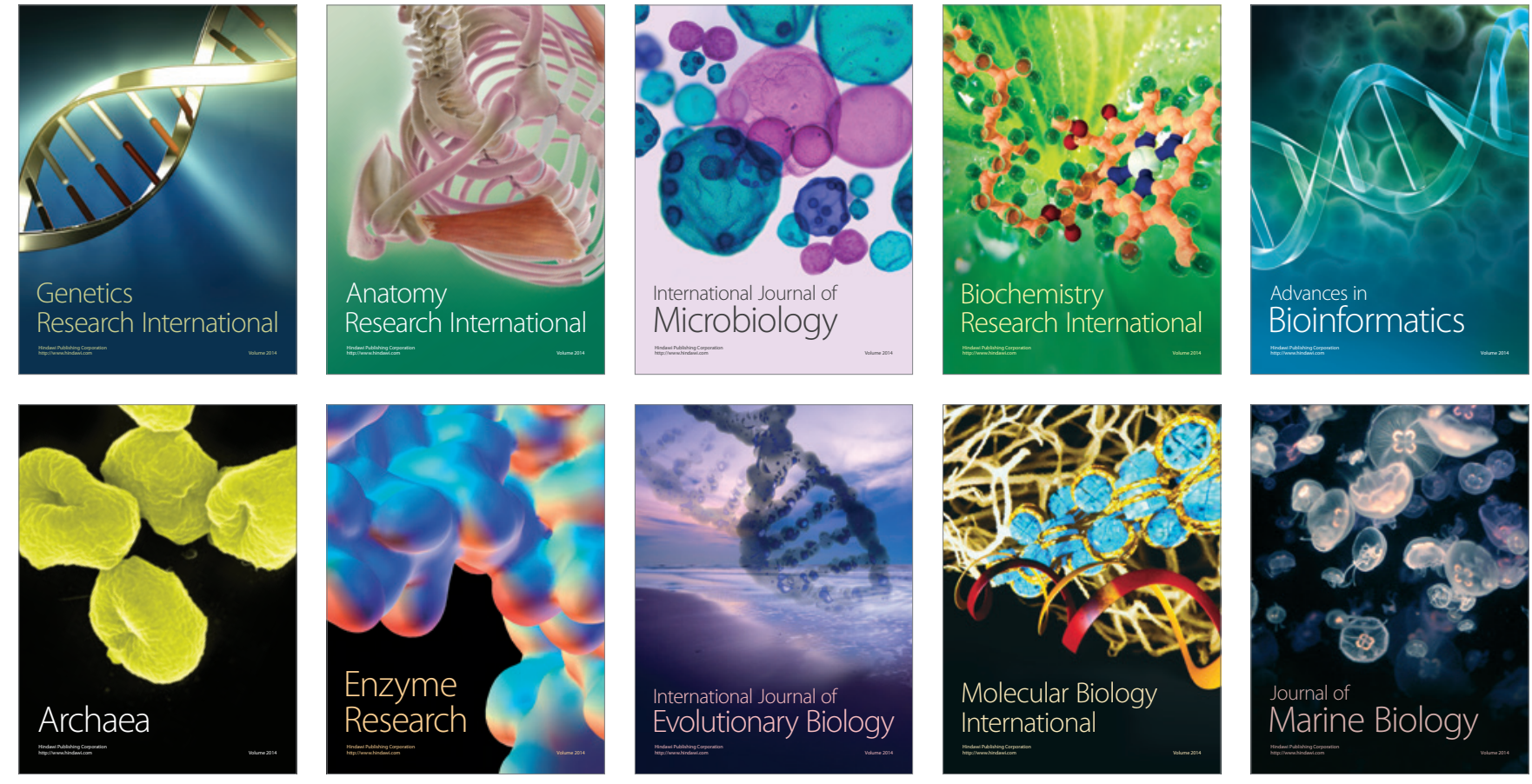